\title{
Protée
}

\section{Le logoclaste. L’entrelangue de Guyotat dans Progénitures.}

\section{Pierre Ouellet}

Volume 29, numéro 3, 2001

Iconoclasmes : langue, arts, médias

URI : https://id.erudit.org/iderudit/030635ar

DOI : https://doi.org/10.7202/030635ar

Aller au sommaire du numéro

\section{Éditeur(s)}

Département des arts et lettres - Université du Québec à Chicoutimi

\section{ISSN}

0300-3523 (imprimé)

1708-2307 (numérique)

Découvrir la revue

Citer cet article

Ouellet, P. (2001). Le logoclaste. L'entrelangue de Guyotat dans Progénitures. Protée, 29(3), 27-37. https://doi.org/10.7202/030635ar

\section{Résumé de l'article}

On dit de la langue de Guyotat qu'elle est iconoclaste ou, plus proprement, logoclaste, puisqu'elle ne se contente pas de détruire les « images » qu'elle suscite, dans son refus de toute mimesis, mais s'immole aussi en tant que langue, à quoi elle substitue cette sous-langue ou cette langue en miettes qu'incarne l'idiolecte où elle se réinvente. Loin d'être la ruine du langage humain, cette parole vive, matricielle et génératrice, qu'on trouve dans Progénitures et dans l'ensemble de son oeuvre, est peut-être la chance inespérée d'une nouvelle histoire de la langue parlée, qui ne s'appuie plus sur la lutte des identités linguistiques (des propriétés ethniques, sociales et religieuses du discours) mais sur la conscience ravivée du mélange dont toute langue est faite. Chaque idiome est « entrelangue » dans la mesure où il s'inscrit entre deux états de langues comme les organes de la copulation et de la génération prennent sens dans un entre-corps, où se mélangent le même et l'autre en un monde " prostitutionnel " que Guyotat décrit en détail pour montrer que l'homme et sa parole n'existent que dans une progéniture ou une pro-genèse sans fin, bien plus que dans une Histoire dont on a dit qu'elle était finie.
Ce document est protégé par la loi sur le droit d'auteur. L'utilisation des services d’Érudit (y compris la reproduction) est assujettie à sa politique d'utilisation que vous pouvez consulter en ligne.

https://apropos.erudit.org/fr/usagers/politique-dutilisation/ 


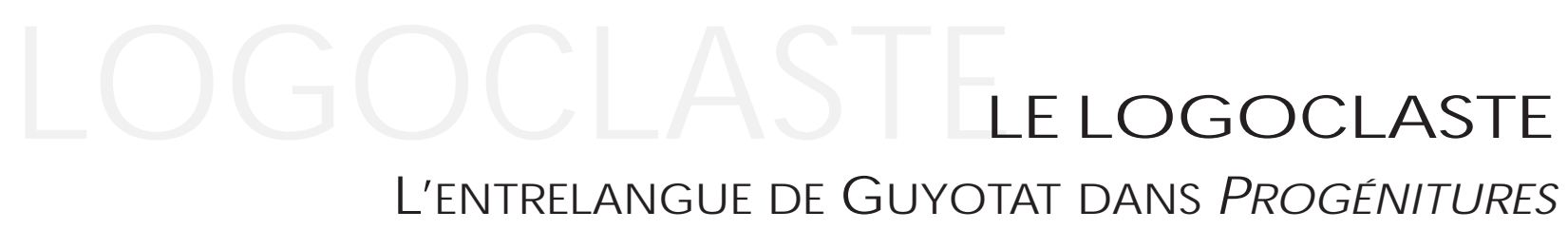

PieRre O u ELLET

Chaque mot, tel qu'il est aujourd'hui dans notre langue, doit restituer la réalité sonore, matérielle, de la chose. C'est un travail, une preuve dont on ne peut pas revenir. Tout cela est tenu rythmiquement. Il faut aussi que la vision tienne, c'est le rythme qui la fait tenir, qui la borde, qui l'étend, etc.

Pierre Guyotat ${ }^{1}$

Voix, grand tremblement. Et le souffle me soulève, il me prend. Et je vais, amer, dans la fière de mon souffle.

Ézéchiel ${ }^{2}$

L'iconoclaste détruit les images par respect pour elles, en quoi il croit et met toute sa foi. L'icône n'est pas pour lui un simple signe, plat, inoffensif, inanimé, une pure apparence plus ou moins trompeuse, mais de l'être au sens fort, un plus qu'être: un apparaître ou une apparition, de l'existence vivante, qui concurrence, par ce surcroît d'être qu'elle incarne littéralement, l'existence même de Dieu, auquel elle risque de se substituer dans l'exercice de l'idolâtrie. Si l'image doit être détruite, c'est qu'elle existe, bien plus qu'elle ne représente et ne signifie: elle est, bien davantage qu'elle ne paraît. Elle vit. Peut-être plus que Dieu, qui n'apparaît pas, lui, se contente d' «être», sans plus, inincarné, inexistant. L'image, elle, est l'incarnation pour l'œil et pour la main - pour le corps tout entier, debout ou à genoux devant elle - d'une âme ou d'un esprit, d'un souffle ou d'une vie qui apparaissent enfin, tangibles, comme si l'âme était un animal et Dieu en chair et en os, comme l'icône est en formes et en couleurs, l'idole en marbre ou en or massif.

Le logoclaste brise la parole parce qu'il y croit, il y croit trop, par excès de confiance et de croyance dans les syllabes et dans les mots, par surcroît de fidélité au verbe en tant que verbe, chair et os sonores à quoi il se fie et se confie, comme aux choses et aux personnes, comme à Dieu lui-même: il est fiancé à la langue, à sa langue, comme on l'est par amour et par désir à qui se donne à soi en échange du don qu'on fait de sa personne en donnant sa parole. La foi seule dans la langue comme force vive, forme vitale, fond vivace, entraîne qui la vit et en meurt à se 
révolter contre la fétichisation et l'instrumentalisation de la voix et de la parole: la langue est, rythmiquement, visuellement, dans l'œil et dans l'oreille comme dans l'âme et dans l'esprit, et ne peut en aucune manière se réduire à un moyen, telle une bouche pour parler, une oreille pour entendre, quand la bouche embrasse, mord, sourit, grimace, mange ou crache, rit ou crie, et que l'oreille nous guide telle une antenne ou tient le corps en équilibre, le protège de ses vertiges. La langue n'est pas pour parler, ni pour penser, ni pour comprendre ou pour communiquer: la langue est pour vivre, pour vivre et pour mourir. Elle est à vivre bien plus qu'à dire ou à entendre. Elle engage la corporalité et la spiritualité tout entières de l'homme, son et sens dans le même organe: l'organe de la parole par quoi l'on souffle, inspire, expire, transpire aussi, car l'âme sonne, résonne, l'âme sue, par tous les creux et les saillies du corps, tous les pores de la peau que le verbe tend sur notre chair pour en faire ce que Valère Novarina appelle La Chair de l'homme ${ }^{3}$, justement, la chair parlante, la chair palpitante de mots et de silence, la parole carnée. Guyotat dit, lui, dans son bas monde, «le rat charogne c'est un medium, comme l'excrément, c'est un pain de conversation" ${ }^{4}$. Car la parole est nourriture: carne et bas morceaux, viande avariée, non pas hostie ou pain bénit, à moins qu'on veuille entendre le mot hostile et le mot otage dans le mot hôte que le verbe incarne et goûter dans le pain qu'on mange en chaque mot le pain qu'on évacue dans son propre souffle.

C'est parce qu'on en mange, du verbe ou du logos, même faisandé, même avarié, qu'on l'exècre et qu'on l'excrète telle une progéniture qui vous sort du corps à tout instant et dont le bruit qu'elle fait en tombant ne cesse de vous rentrer dedans, ensuite, nourriture de sens et de non-sens qui roule dans votre bouche comme roule sous votre pas cet air en boule que votre souffle expulse de votre ventre pour qu'il marque votre chemin dans le monde et dans le temps:

d'entr', en rue, femell', mâl', pubèr', non-humains, t'brosser, vagin, mott', l'chiambranl' rouj' mouchiassats aux trous, rapaç', assoupis en dépouill', / d'quall'bouch, d'entr'saillies fil', d'commis mâtr' renfiaulés rerougie, l'mâl' la gorj' son épaul', la femell' sa faç' d' faç' [...]. 5

Oui: de quelle bouche d'entre-saillies peut encore sortir une langue? Une langue qui ne s'écrit plus dans son sens, qu'elle a perdu ou expulsé, mais dans son souffle, même arrêté, son air asphyxié, son air étranglé: les apocopes et les aphérèses abondent dans Progénitures, qui disent la chute d'un son ou d'une syllabe en bas du mot, à la fin comme au début - apokopè: retranchement, amputation, abolition et, par extension, arrêt brusque de la voix; aphairèsis: action d'ôter, d'enlever, de soustraire, de séparer et, par extension, de couper brusquement la parole. On nous a coupé la parole, nous l'a mise en pièces, corps amputé dont les morceaux gisent dans notre langue comme autant de membres et d'organes éparpillés d'une progéniture mâle ou femelle qu'on ne reconnait plus: «bouch'», "gorj'», «épaul'», «faç' ", «vagin», «mott'», tous « rouj' mouchiassats aux trous», dit Guyotat, précisant ce que parler veut dire, désormais, pour le "non-humain putain" à quoi nous sommes ramenés en ces temps durs d'extrême besoin: «l'craier crachier d'dans leurs poangs, / s'reempouangner l'entr'-jiamb'»6, l'entre-iambe ou l'entre-langue, entendons-nous, qui savons bien qu'on ne parle pour vrai qu'en "crachiant» les mots telle une semence disséminée, un sens errant, ou égaré, et en tenant dans son poing l'organe secret de cette parole qui donne naissance à une vision mort-née.

\section{L'AVALEMENT DES LANGUES}

L'Histoire nous a vieilli la langue, nous l'a gâtée, nous l'a pourrie, mais on n'a pas cessé de l'avaler pour autant, de la dévorer, puis de la régurgiter, presque dans le même mouvement. L'Histoire selon Guyotat aura donné naissance à une espèce d'être entre les règnes, comme une langue entre les langues: l'êtreputain, le non-état putain, qui est entre l'homme et l'animal, le règne du sans-règne, le non-être de l'asservi, pourtant proche du divin, «des figures angéliques», «sales et secouées par les accouplements» ${ }^{7}$, des anges animaux, des animanges. 
Les putains, dit Guyotat, sont comme des paroles divines incarnées, d'une certaine façon. Incarnées momentanément, mais elles durent tout de même parce que les paroles s'engendrent... progénitures de paroles; c'est aussi comme ça qu'il faut comprendre le titre de l'œuvre. Et là, la nourriture de base, et même la seule nourriture, c'est la charogne; la charogne de rat. 8

La boucle est bouclée: nourriture avalée, progéniture expulsée. Autrement dit: ange mangé, chié putain. Le verbe n'échappe pas à ce cycle dans lequel l'Histoire enferme, malgré son apparente linéarité: tout logos ingurgité, à force de fidélité au verbe adoré, qu'on veut à tout prix s'incorporer, incarnant Dieu dans sa pauvre chair d'homme, sa chair à paroles et à cris, finit un jour par se vomir en alogos, mots et choses entremêlés dans la même glèbe morte, qu'on porte sur son visage autant que dans sa bouche: "toute cette crasse qu'il y a, intense, sur toutes ces figures, vient de ce que la nourriture est principalement de cet ordre» ${ }^{9}$ - et la progéniture autant, qui souille le parturient, comme la charogne le mangeur, sa semence le putain.

Parole-Nourriture: Semence-Progéniture. Voilà le carré dans lequel se meuvent charognes et putes, tous deux «verbes», tous deux bas logos, paroles du bas. Guyotat dit des rats qu' «ils sont la nourriture du verbe putain " 10 - le putain étant "parole divine incarnée», «parole qui s'engendre» de toute éternité -, puis il ajoute que le rat n'est pas seulement la nourriture de base, la nourriture du bas, mais aussi et plus singulièrement

[...] un élément de base du son du livre, ce craquement d'une nourriture entre les dents des putains mâles ou femelles [...]; c'est le craquement des os, de la chair, de la peau, de la queue, du museau entre ses dents, dans ses mâchoires qui signale la présence d'un individu putain. 11

Et ce craquement d'os fait entendre «la volonté de parole» des asservis divins qu'incarnent ces bouches à nourrir et à cracher, ces bouches à vivre et à mourir que sont les putains hommes ou femmes dans leur parole ouverte, toujours prête à avaler et à régurgiter n'importe quoi, semence ou rat, progéniture sans fin. Partout dans la Bible YWHW s'écrie d'un seul et même souffle: "je te bénis, je multiplie ta semence", comme si la parole, dans son état divin, sacrait et engendrait du même coup, avec ce pain de conversation qu'est l'excrément, le rat ou la charogne, ingurgité par le putain qui en fait son âme, son souffle, son rythme, et ce vin de procréation qu'est la semence répandue dans tous les corps par l'ange prostitué qui se multiplie, proliférant au rythme de la chair faite chair encore et encore, parole proférante qui porte en elle et met au monde l'humanité dans toute sa lignée. Écrire, parler :

[...] nourrir mes ptiots mes épouz' renourrir mes frèr' soeurs pèr' mèr' leurs pèr' mèr', leur changer chair et os sang reprocréer leur descendanç'?, desintegrés - fracas, lueur, relent m'evailler?, non!-, d'quall' croup' m'ebrouer d'ma semenç' au ventr' la faç' ma faç' la bouch' m'mâchier d'quà d'l'empeign' l'tir' d'l'égout l'fouler aux mâchoir' blanch' [...] ${ }^{12}$,

car toute parole revient à ça: ébrouer sa semence, se «mâchier» jusqu'à l'empeigne, fouler l'égout aux mâchoires blanches, bref ne plus connaître le terme ni le début, confondre les bouches, les embouchures, l'origine originante et la fin finissante, toutes deux dans la même «bouche que veux-tu» qui avale tout, sachant qu'elle peut à tout moment le «recrachier» en mots et en toutes sortes de génitures, verbales mais sexuées, animales mais angéliques, putains dans tous les sens, c'est-à-dire passant d'une main à l'autre, d'une bouche à l'autre, comme on s'échange du sang ou de la salive pour mieux s'unir et s'embrasser, à défaut de communiquer. Il n'y a plus de livres, plus de langues, mais une matière que l'on se passe de bouche en bouche dans les baisers et les morsures, qui creusent leurs cavités, les vides, les gorges, ces vases communicants qui permettent au sens liquidé, au sens liquéfié, de circuler encore dans les membres et les organes du non-être humain que nous sommes devenus, nourris de mots et de silences qui tuent. On a dit de la langue de Guyotat qu'elle était tordue, au sens où l'on parle d'un linge qu'on tord, comme Chaïm Soutine fait de ses toiles, non pour 
[...] en exprimer le liquide dont il est gorgé, [...] non pour

dégorger, souligne Daniel Klébaner, mais pour faire gorge,

produire l'organe tortueux à travers lequel la voix fait effort pour

faire passer le souffle, résonne en tant qu'elle racle et lutte contre

les replis et les nodosités de la chair. ${ }^{13}$

La langue tordue fait gorge dans une gorge, où s'accumule le sens fondu et confondu, qui s'y dépose en cailloux, caillots, calculs, nodules, petites nodosités dans la pure cavité qui les recueille dans son vide pour mieux s'en gorger. Le livre est une autre bouche dans sa bouche où résonne la voix de l'autre tel un écho dans le fond d'une grotte, un bruit d'eau qui coule dans une gorge, le goutte-à-goutte des mots qui tombent du palais et se renfoncent dans le larynx, où l'âme suffoque, sa parole blessée sous les coups de glotte que lui assène le pouls du Verbe.

Souvenons-nous d'Ézéchiel, à qui Dieu s'adresse en ces mots:

Fils d'humain, ce que tu trouves, mange-le! Mange ce volume et va, parle à la maison d'Israël! [...] Fils d'humain, nourris ton ventre, remplis tes entrailles, avec ce volume que, moi, je te donne.

- ce à quoi le prophète répond, non par les mots mais par sa bouche qui bée, son corps béant: «j'ouvre ma bouche et il me fait manger ce volume. [...] Je mange et c'est dans ma bouche comme une douceur de miel» ${ }^{14}$. Bouche et ventre communiquent mieux par le volume de Dieu - logos divin fait miel, lait et semence, pain et viande, carne, rat ou rot: progéniture verbale qui s'enfante infiniment dans l'éternité de la faim et de la soif, de l'amour et du désir - que ne communiquent entre eux l'homme et l'homme par le langage désincarné de l'Histoire. Certes, le miel a goût de fiel, désormais, et le volume de Dieu ne tient plus dans la bouche de l'homme, pleine d'excréments dont il a fait son pain quotidien, son pain de conversation, mais tout le dit haut et fort: la parole est d'or, la parole est chère, la parole est d'air, la parole est, et le non-être dans lequel on plonge depuis l'Histoire ne peut se perpétuer, progéniture sans foi ni loi, que par cet être de surcroît qu'incarne le verbe aspiré puis expiré en soi, le volume avalé, qu'on restitue à Dieu comme à autrui dès lors qu'on parle ou qu'on écrit, non pas la langue elle-même, logos à jamais consommé, mais l'entrelangue qui nous revient sur le palais, entre les lèvres, au fond de la gorge, ces entrailles de la parole où l'on sent le rat dans le mot, la charogne en quoi le verbe se fait, le putain dans l'homme et toute cette progéniture qu'on appelle verbe ou écriture.

L'iconoclaste avale l'image comme si c'était Dieu lui-même, son air, son souffle, son âme: il la détruit en la mâchant, la réduisant en pièces pour que seul reste le pur Imaginé, mais qu'il reste entier dans son être, inapparent, entier dans son rythme, son pouls, sa vie, même si l'image de son visage est en morceaux, méconnaissable. Le logoclaste avale sa langue comme une queue de rat dont le craquement des os et de la peau révèle dans sa gorge et ses poumons la voix de Dieu déchiquetée, disséminée, telle une semence multipliée, une progéniture sans nom, une pure et simple prolifération, de la vie dans de la vie encore, de la vie après la vie, de la survie: une pure profération, qui pousse devant, au loin, plus loin (pro-ferre: se porter au devant), une écriture, une prophétie.

\section{LE SANS-NOMBRE, LE SANS-FIGURE}

Les langues ont plusieurs sources, comme les cultures, comme chacun de nous. La langue sourd de partout, jamais d'un seul sol ou d'un territoire unique. Pas de loi du sang, chez elle, pas de loi du sol, car elle n'a pas de paternité, pas d'origine fixe, et elle n'a pas de lieu natal, pas de maison mère: elle coule orpheline comme le sang anonyme des peuples, humains ou bien putains, en alluvions et confluents de toutes sortes, puis elle va sans biens et sans abris tel un immense glissement de terrain qui serait aussi l'imperceptible tremblement du temps où aucun sol ne tient, aucune histoire non plus, aucune généalogie qui ne se dissolve aussitôt en multiples afflux et reflux, en affluences sans fin. La littérature retrempe la langue dans ces innombrables sources d'où elle rejaillit sans cesse sous une autre forme, souvent méconnaissable. Toute l'œuvre de Guyotat fait 
entendre cet éternel ressourcement de la parole dans l'étrangeté du monde d'où elle vient et où elle revient, jamais chez elle, toujours à l'étranger. Ses livres sont écrits non dans une langue mais entre les langues qui font du français un idiome vivant, progéniture verbale née de l'accouplement des voix et des parlers, des accents et des patois, des sabirs et des créoles les plus singuliers. C'est une langue qu'on ne reconnaît pas d'emblée parce qu'elle échappe à toute identité: ce n'est plus une langue nationale, ni une langue universelle, et encore moins une langue personnelle ou idiolectale, mais la langue du devenir de l'espèce, la langue en mutation - l'entrelangue de l'interrègne propre au temps des asservis, du non-état putain. Dans l'entrelacs de cette langue, «les mots lèvent les pensées comme les chiens lèvent les lièvres " 15 , dit Guyotat: le sens est proie, car tout est chasse et l'homme dans ce carnage court et aboie, la langue pendante, pour être enfin de la curée, goûter aux restes et aux abats, aux bas morceaux que l'histoire nous laisse, verve insensée, carne arrachée, entrailles et entrelangues que seul un museau d'homme peut démêler, s'il a le flair des mots, les dents du verbe bien aiguisées.

Il y a plusieurs manières d'être entre les langues, de vivre et d'écrire entre deux langues ou entre cent. Il y a la transhumance, le déplacement: les transfuges d'une langue à l'autre, comme Nabokov ou Cioran, passés du russe ou du roumain à l'anglais ou au français, dans une sorte de déménagement ou de grand dérangement verbal où l'on quitte sa demeure pour une autre, avec ses pénates. Il y a l'alternance, la «biphasie», comme on parle de bigamie, de ceux qui passent d'une langue à l'autre selon l'œuvre ou le type de textes envisagés: Beckett, qui va de l'anglais au français ou inversement, selon qu'il écrit du théâtre ou des récits, Brodsky, qui fait ses poèmes en russe, ses essais en anglais. Il y a le collage ou la juxtaposition, la collision et la collusion des langues entre elles au sein d'une même œuvre, comme dans les Cantos de Pound, où le chinois, le grec, le latin, l'italien et l'anglais se voisinent d'autant plus près que leur grammaire et leur rythme semblent éloignés, comme dans l'image poétique que Reverdy définit par le degré d'étrangeté des termes qu'elle rapproche. Il y a l'agglutination ou la superposition, comme dans le Finnegan's Wake de Joyce, où les mots eux-mêmes peuvent être composés de radicaux et d'affixes provenant de plusieurs langues plus ou moins apparentées, et où la syntaxe, dans ses structures les plus profondes, peut obéir à des lois qui régissent des langues ou des dialectes incommensurables. Il y a encore le surgissement, au sein d'une langue comme le français, d'une non-langue ou d'une langue qu'on dit inventée, comme chez Michaux, avec ses néologismes aberrants, chez Artaud, avec ses glossolalies et ses lalations, chez Gauvreau et son exploréen, chez Dotremont et ses logogrammes. Et puis il y a les différentes formes de diglossie ou de code switching, qu'on connaît bien au Canada et au Québec, où l'on passe volontiers de l'anglais au français chez de nombreux poètes qui témoignent d'une situation géopolitique et géoculturelle caractérisée par le bilinguisme. Mais l'entrelangue de Guyotat renvoie à une tout autre façon d'être entre les langues, d'être parmi elles dans sa langue même, non plus en les juxtaposant ou en les superposant, ni non plus en les alternant entre elles ou avec de la nonlangue, mais en les vivant, toutes, mortes ou vives, au sein d'une même langue désormais sans identité, inidentifiable, non reconnaissable comme LA langue française en personne, centrale, dominatrice, coloniale ou impériale. On a affaire à une langue blessée, meurtrie, on pourrait dire torturée, dont les violences qu'elle aura fait subir à l'autre se retournent toutes contre elle, dans une violence encore plus grande, parce que provenant des violentés, des cris et des pleurs des violentés, de leur colère et de leur détresse, de leur souffrance en condensé. C'est une langue qui se survit, une langue d'après sa fin, où elle retrouve sa nudité, comme au début: une nudité souillée, toutefois, qui porte l'habit déchiqueté de notre humanité déchue, de notre putanité parlante et déparlante dont le sens a été perdu, vendu. Une langue qui survit à son âme morte ou à son centre absent, défait par l'Histoire ou par le Temps, en se 
déplaçant continuellement à sa périphérie, sur ses limites les plus extérieures, qui en deviennent le cœur, bientôt, le tout premier moteur, celui qui la fait battre et marcher encore et encore, bien qu'elle soit épuisée.

Écrire entre les langues n'est pas comme être assis entre deux chaises, la jambe gauche dans une langue et la droite dans l'autre, tout le reste de sa personne suspendu dans le vide: une moitié de soi parlant l'anglais, l'autre le français, leur rencontre reposant sur du vent, sur un pur néant. La diglossie n'est pas l'entrelangue, mais la représentation de deux absences de langue, chacun des deux idiomes se cachant dans l'autre plutôt que de s'y révéler, de s'y mettre à nu, dans ses blessures, ses cicatrices, ses cernes et ses rides, ses signes de vieillissement prématuré, dans son extrême fragilité causée par sa longue exposition à l'histoire, au monde, au temps. On dissimule ainsi la difficulté qu'on a d'être tout entier dans la langue, en restant simplement entre elles, dans l'entre-deux, protégé des dangers que représente le fait de vivre et d'écrire l'altérité au cœur même de sa langue mise à nu, exposée, surexposée à toutes les autres, et d'y être intégralement, soi-même exposé au principe de cette nudité, non plus réfugié entre deux moitiés de langue qui ne donnent jamais de la langue mais de la «milangue", toujours, où le mélange annule ce qu'il mixe plutôt que de l'amener à procréer et à se procréer. Car une langue, quelle qu'elle soit, se crée et se recrée par et dans toutes les langues qu'elle comprend, réellement ou virtuellement, et en son propre sein, si je puis dire, même si elle n'a ni dehors ni dedans.

Prise dans sa profondeur historique - son véritable sein, son authentique giron, où elle progénère au sens propre -, une langue parle toutes les langues qui l'ont faite et qui l'ont défaite, qui la refont et la redéfont. C'est dans son histoire la plus intime et la mieux partagée - celle qui la retourne sur sa mémoire (ce qu'elle retient de son passé le plus lointain) et la détourne vers son imagination la plus vive (ce qui la projette dans son avenir le plus incertain) - qu'une langue rencontre en elle cette entrelangue d'où elle vient et où elle va, toujours mouvante entre deux états, deux états de langue, comme on dit, qui ne sont jamais qu'une phase de son évolution, un épisode de son histoire, une tranche de vie. On ne peut avoir accès à la langue qu'en feuilleton: elle ne paraît jamais d'un coup, mais se donne par fragments dans la suite des temps, elle se donne en pièces, logoclaste ellemême puisqu'elle ne se présente jamais que découpée, en coupe, comme sur l'étal du boucher, dirait Guyotat, nulle part dans son intégrité. Toute langue est chronique, non seulement parce qu'elle dure longtemps, comme lorsqu'on parle d'une maladie chronique, mais surtout parce que son existence est intimement liée au temps qu'elle prend pour se montrer ou se proférer, se révéler et se prolonger ${ }^{16}$, dans ses transitions, état par état, partie par partie, chacune n'étant qu'une entrelangue, vivant dans l'entretemps, tendue par la mémoire vers son passé et par le rêve vers son avenir, qui ne peuvent se vivre qu'au cour des autres langues, qu'avec les autres langues, dont elle s'imprègne au plus profond pour se connaître dans son intimité, où elle se trouve autre, toujours, jamais identique à elle-même. Et l'autre, c'est soi aussi, dans l'étrangeté de sa vie, son étrange mortalité, cette évidence ou ce mystère: jouissance et souffrance indémêlées, quand «le travail de la langue», comme celui de la parturiente et de l'engrossée, de l'accouchante et de l'ensemencée, «c'est du plaisir ou c'est de la souffrance, ou pire, les deux en même temps" ${ }^{17}$, en leur énigme. Dans Viure, Guyotat écrit: "ce qui est de l'ordre du mystère ne peut s'exprimer dans une langue commune» ${ }^{18}$; il précise aujourd'hui: «Seule la langue du chant permet en même temps de faire passer le mystère et de renforcer l'énigme; de les rendre encore plus troublants» ${ }^{19}$, parce que

[...] la langue d'un auteur, c'est sa voix, c'est ses poumons qui hui sont donnés, ce n'est pas la capacité pulmonaire, bien entendu, c'est la vitesse innée du coeur, la vitesse de la réaction du cœeur enfant au monde qu'il découvre 20 - [...] la langue d'un auteur, ajoute-t-il, grandit avec lui; elle fait son chemin dans son cerveau, dans son coeur et dans son corps. [...] La vie et la langue avancent ensemble. L'une fatigue l'autre, l'autre ranime l'une. 21

L'entrelangue est entrevie. Entre naissance et agonie, entre vie et mort, elle est survie: lutte, angoisse, 
agônia. Destin du non-état-putain. L'entrelangue est un état interne, une vie intérieure à la langue ellemême et au poumon qui la respire, à l'âme qui la souffle, à la vie même de l'air dans notre propre chair, qui y circule comme un deuxième sang, plus rouge que le premier: «rouj'mouchiassats... renfiaulés rerougi» - Guyotat écrit, parlant de son entrelangue de toujours: "c'était une langue interne, c'était une voix : un destin, c'est ça ${ }^{22}$. Vivre et mourir en langue et en rythme, comme on dit en chair et en os, logolâtre et logoclaste d'un seul et même souffle, qui ravive la flamme des mots et puis les mouche, en une seule profération, une même fiction verbale qui fait être ou donne naissance puis fait disparaître ou donne la mort, don ou contredon qui procrée et détruit comme on respire, inspirant et expirant à pleins poumons, à pleine bouche, à tue-tête. Vie et mort réunies, en une même sur-mort, une même sous-vie, destin du verbe putain entre boulimie jouissante et souffrante anorexie, ascèse et excès dans l'accès brusque à l'être qui est décès du temps, mort de l'Histoire, effacement lent de toute frontière entre dehors et dedans, soi et autre, langues et non-langues, images et non-images.

L'entrelangue n'est pas hors de la langue, extérieure à telle et telle langues, par exemple, dans le vide qui les sépare, dans l'interstice ou le hiatus qui donne l'impression qu'on aurait affaire à deux langues distinctes, ayant leur identité propre. Elle est au cœur de toute langue, c'est elle qui en fait battre la vie même si ce doit être dansl'arythmie, comme toute image n'apparait que sur un fond d'iconoclasmie, fond d'or, fond noir ou fond perdu, infigurable et nu, comme la vie elle-même sur son fond de mort et toute apparition sur la lisière du disparu. Ce sont, entre autres, les langues mortes dans les vivantes qui leur permettent de croître, de procréer, de se multiplier à travers la néologie, l'invention verbale fondée sur la mémoire des langues disparues, l'innovation ou la futurition lexicale s'appuyant sur le plus lointain passé, même oublié. Le français est grec, latin, franc, gaulois, germain, normand, provençal et occitan, quoi d'autre encore, et il est romand, wallon, québécois, créole, sabir, etc., tout en étant surtout et à chaque fois une puissance de création par entrelangue qui nous donne à la fois Rutebœuf et Chrétien de Troyes tout comme Jarry et Rimbaud, Artaud, Michaux, et aujourd'hui Prigent, Novarina, Guyotat. On n'a qu'à mettre côte à côte ces paroles-là pour voir et entendre qu'une langue n'est pas une chose, avec son identité propre, toujours reconnaissable, dans ses contours précis, bien définis, mais un milieu dans lequel on vit, par lequel on vit et survivra, comme l'air que l'on respire, l'espace où l'on se meut, le temps où l'on mûrit puis vieillit, changeant de place d'époque en époque et du même coup d' "identités", si une telle chose existe, je devrais dire persiste, subsiste. La langue est un milieu, oui: c'est un cœur et c'est un monde, comme dit le mot lui-même, qui désigne à la fois un noyau ou un moyeu, central, et un entour ou une périphérie, qui l'environne et tourne autour. C'est un centre et un mitan, mais c'est aussi un cadre et un contexte, une atmosphère ou un climat, comme on parle de biosphère ou de biotope, par exemple, de milieu urbain ou de milieu humain. Mais le milieu, c'est encore le mi-lieu, l'entre-lieu, le lieu médian, l'intermédiaire, l'entre-deux, ce que nomme l'entrelangue qu'incarne toute langue en son cœur et en son décor, sans cesse fluctuant, passant d'un état à l'autre, jamais stationnaires, toujours transitoires, impossibles à identifier autrement que par cette puissance de changement et cette force génératrice ou génésique qui caractérisent toute langue vivante, toute langue en gésine, qui accouche d'elle-même à tout moment, qui accouche d'elle-autre, devrais-je dire, se donnant naissance comme une autre et une autre encore, de Villon à Guyotat. La langue est entre, essentiellement, et c'est un pur pléonasme de parler d'entrelangue comme je le fais ici.

La langue ne s'arrête jamais, comme on arrête un sens ou une idée. On ne peut la définir parce qu'elle n'est pas finie, elle n'est jamais finie. Comme la Partie de Beckett, qui n'a pas de fin et n'en aura jamais: "fini, c'est fini, ça va peut-être finir " ${ }^{23}$, dit Clov, dans sa voix blanche, son regard fixe, mais il sait bien qu'on est encore au commencement, toujours, que la partie 
n'est jamais jouée, que la partie n'est pas un tout, dont on peut dire au bout du compte: c'est ma vie, mon histoire, ce que je suis. Ce n'est jamais fini parce qu'il y a toujours à dire, trop à dire, même quand il n'y a plus rien, parce que la langue continue de parler en nous, même quand on se tait, qu'on voudrait se taire, parce qu'on se sent fini, ou qu'on sent l'Histoire finie, qu'on sent la finitude de l'homme et la sienne même, comme on sent son propre corps vivre et mourir dans le même moment, et qu'on se sent mal, soudain, au bord de l'évanouissement. Hamm et Clov, tous les personnages de Beckett, en fait, comme ceux de Guyotat, qui parlent comme ils forniquent, sont bavards à force de n'avoir rien à dire, rien à dire d'euxmêmes ou par eux-mêmes, si peu sûrs qu'ils sont de leur existence ou de leur identité: ils s'en remettent tout entiers au bavardage de la langue en eux, entre eux et autour d'eux, à l'infini bavardage d'une langue qui ne mourra pas avec nos derniers mots, qui ne s'éteindra pas dans notre dernier souffle. La langue nous survivra et nous le savons. Elle survit aux civilisations: on peut lire le hittite et le sumérien comme le français et l'anglais, si on s'y met, mais on ne ressuscitera jamais le monde où ces langues furent parlées à l'origine, parce que ce monde est un monde fini, achevé. Chaque moment du temps finit par rejoindre cette origine perdue: chaque instant va mourir en elle et avec elle, disparaître dans un passé bientôt immémorial, mythique et ancestral, préhistorique. Alors que les langues, celle d'Homère ou de Virgile, celle de Montaigne ou de Racine, survivent dans la langue que nous parlons et qu'on parlera bien après nous. La langue a un destin, nous n'avons que nos vies.

\section{UNE FIN QUI N'EN FINIT PLUS:}

\section{LA LANGUE DANS TOUS SES ÉTATS}

La langue est cet infini ou ce non-fini qui nous permet de faire face à notre finitude. Elle est ce lieu de rencontre avec l'Autre, en grand nombre, en très grand nombre - dans la multitude passée et présente qu'elle porte et qu'elle transporte depuis la nuit des temps et les confins du monde -, cet autre multiple, donc, indénombrable, non totalisable, qui nous permet de faire face à notre identité perdue depuis toujours, pas plus reconstituable que l'origine, c'est-àdire à cette impossible et improbable image de soi en soi à quoi la langue comme lieu du pullulement des sens supplée dans notre mémoire et notre imagination. Ce que Guyotat énonce sous la figure de la profusion: rats, mouches, vermines, crasse, poussière, peuples, paroles, proférations, déglutitions, défécations - images, toutes, d'un incommensurable, qui dépasse toute commune mesure. La langue n'a pas d'identité propre parce qu'elle naît potentiellement de langues sans nombre, mortes ou vivantes, et qu'elle croît en se greffant virtuellement à d'innombrables langues présentes ou à venir. C'est un modèle de société, cette socialité première des mots et des paroles qui composent une langue, non pas avec du même, de l'identique, mais de l'autre et de la différence qui la nourrissent du dedans - qui ne l'envahissent pas, ne la conquièrent pas, ne la parasitent pas non plus, ne la phagocytent et ne la cannibalisent pas davantage, mais l'alimentent et la sèvrent du même coup, la désaltèrent et l'altèrent en même temps, depuis son fond et ses tréfonds, telle désinence étant latine, telle construction grecque, tel affixe germain ou anglosaxon, sans que l'ensemble cesse d'être telle ou telle langue, le français, par exemple, dans sa réalité nécessairement mouvante, jamais identique à ellemême. La langue est un mécanisme fini, bien sûr, et en ce sens terrestre, humain, bien trop humain, immanente à notre existence mondaine et historique, mais elle produit de l'infini: de nouvelles phrases à chaque fois, qui ne reviendront jamais, qui feront place à d'autres, dans une génération sans fin, une incessante "progéniture». En ce sens la langue est du fini infinitisant, ou, plus proprement, de l'infini immanent, de l'infini bas, pour le distinguer des absolus et des transcendantaux, qu'on lui associe généralement et qu'on place toujours très haut ${ }^{24}$.

La langue est infinitisante parce qu'elle a à voir avec l'indéfinissable, l'inidentifiable, l'indénombrable, le pullulement que Guyotat, encore une fois, figure sous la forme de nuées de mouches ou de hordes de rats, 
bref de tout ce qui prolifère à toute vitesse et par le bas, par le plus bas. Ce qui rappelle encore les mots de Clov: «les grains s'ajoutent aux grains, un à un, et un jour, soudain, c'est un tas, un petit tas, l'impossible tas " ${ }^{25}$. La langue, c'est ce qui prolifère en tas, par tas, comme les mouches, comme la vermine et les rats, non pas grain par grain, un à un, car il n'y a plus d'un, plus d'unité: la force pullulante et infinitisante de la langue comme pure gésine ou genèse de l'espèce l'a fait éclater en un millier d'existences autres, tout autres, qu'aucun Dieu ni aucune transcendance ne peut unifier ou rassembler. Le verbe être est une copule, diton, et il abonde dans notre langue, où il lie et relie les mots en phrases, renvoyant à la prolifération des accouplements, des couplages et copulations qui adviennent dans notre monde. Le verbe être est «entre», toujours, parole de l'entremise, de la mise entre, de l'entrelangue. Mais le verbe putain ne donne le jour à rien, il donne la nuit, plutôt, dans la mesure où l'ouvrier qui rentre la nuit, après sa journée de nonvie, rentre dans la nuit, dans sa nuit, dans «la possibilité de remanger, de re-désirer, de continuer de vivre, de se mouvoir en état de copulation, de s'épancher au propre et au figuré; le tout en état de parole intense» 26 - il rentre dans la survie. Guyotat nous le rappelle:

[...] les putains ne "procréent» pas, ils «reproduisent»; ils ne «naissent» pas, ils sont "mis bas»; ainsi, précise-t-il, j'ai eu du mal à choisir en fin de compte, à cause de sa charge de réel, le terme de «maître-naisseur» [qui est un terme vétérinaire], j'aurais pu écrire «maître-met-bas» 27 ,

- voilà : la langue met bas, l'auteur est maître-naisseur. Le verbe putain est de l'infini à profusion, de l'infini animal, de l'infini de «bêtes de somme», comme dit le mot latin veterina: "propres à porter les fardeaux". C'est en ce sens que la langue est porteuse: une bête de somme qui porte le fardeau de l'homme et le met bas, très bas, où il pullule et s'infinitise comme la cendre et la poussière, comme le tas, l'amas. Du transcendant vétérinaire: des "anges de somme», tous ces putains porteurs de l'entrelangue, de l'entrechant, qui ne reproduit que leur mystère, celui de la copulation, de la mise bas, de la profération proliférante, de l'infini sexué, langué, gorgé, que l'homme porte en lui comme un trou immense où rassasier toutes ses soifs, tous ses désirs.

La langue est l'infini humain, trop humain: de l'infini putain. Elle pousse l'homme à prendre sa revanche sur la divinité en lui donnant la possibilité de tout créer et recréer à chaque instant, de tout détruire aussi, fiat lux verbal qui lui permet non seulement de séparer les eaux d'en haut et les eaux d'en bas à tout bout de champ mais encore de les refondre, de tout recommencer sens dessus dessous ou sens devant derrière:

recul'!, m'redrass, reins, croup', dos, cou, l'long mon devant, [...] la saillie de reappropriation, [...] l'verb' m'manquer, ah Diou!, à-devant ta gorj' muett', t'evacuer en ton vagin, mon pèr', avec ma vain' semenç', ma moell', mon cerveau!,

comme dit Progénitures dans ses derniers mots ${ }^{28}$. La langue, c'est de l'infini actuel, dirait le mathématicien, de l'infini réel: elle fait apparaître un nouvel espace entre les espaces les plus contigus, de nouvelles phrases entre les phrases les plus rapprochées, une parole inédite entre les paroles les plus voisines ou les plus communes. Chaque phrase qu'elle nous donne de prononcer est à chaque fois nouvelle, inédite, imprévisible, même si on l'a déjà entendue dans un autre cadre, un autre contexte, un milieu autre qui lui aura du même coup donné un autre sens ou une autre tonalité. Chacune s'inscrit parmi un nombre infini de phrases possibles qu'on aurait pu énoncer à sa place et vers lesquelles elle ne cesse jamais de faire signe parce qu'elle dit aussi ce qui n'est pas dit, qui lui sert d'horizon, fluide et mouvant, le non-dit qu'elle implique lui servant d'appui fictif, friable, fragile. Une phrase est la pointe d'un iceberg à la dérive: c'est la partie visible ou audible d'un monument immergé dans l'histoire et qui se meut lentement à la surface du temps, changeant de lieux et d'orientations au gré des vents et des courants. Des courants de fond, devrais-je dire, qui échappent à l'œil nu, à l'oreille nue aussi. Il faut savoir entendre la langue dans ses bruissements et ses craquements de surface, qui 
remontent de ses tréfonds, il faut savoir en observer les brèches et les fendillements, les fêlures et les ruptures, qui apparaissent sur ses flancs les plus enfouis, pour pouvoir sentir et ressentir les tressautements et les ébranlements que le temps long de l'histoire ne cesse de faire subir à cet immense monument vivant mais troué d'air, de vide, et parcouru de cicatrices, de rides et de gerçures, qu'on appelle une langue, une langue vivante mais peuplée de langues mortes et non encore nées, hantée par des langues passées et des langues à venir qui sont en elle comme des fossiles réanimés ou des matrices sans cesse réactivées.

La langue est un mécanisme génératif, nous rappelle le linguiste: elle génère, elle progénère, elle se reproduit au sens biologique du terme. Elle se donne à elle-même naissance en proliférant à l'infini en phrases nouvelles et inédites, puis en muant et se transmuant en dialectes, idiolectes, patois, jargons, créoles, pidgins ou sabirs de toutes sortes, au long de son histoire plus ou moins mouvementée, de ses dérives dans le temps océanique où elle ne cesse de flotter ou de surnager même quand elle nous paraît sur le point de couler. En ce sens, la langue est une progéniture, comme le dit et le répète Guyotat. Mais la langue nous donne aussi naissance: elle nous permet de nous reproduire de génération en génération à travers sa transmission et sa transformation, par la mémoire qu'elle garde de l'espèce et de la culture, de l'histoire et des communautés, mais aussi par l'imagination qu'elle nous confère face à notre avenir comme sujet parlant. Elle nous fait naitre au monde, par les mots et les phrases qui nous en ouvrent l'accès, toujours plus large et plus profond, plus infini, et elle nous fait naître à l'autre, par les possibilités d'échange, de contagion réciproque, qu'elle permet dans la parole adressée ou la parole dialoguée - car les mots nomment les choses et le monde, bien sûr, mais ils appellent aussi, les autres, quiconque et tout un chacun. De sorte que nous sommes aussi sa progéniture, à cette langue que nous parlons et à laquelle nous nous allaitons, nous nous abreuvons, nous nous nourrissons pour vivre et pour survivre en tant qu'humains, c'est-à-dire en tant qu'êtres parlants, espèce verbale qui ne trouve son salut que dans le giron des langues, dans leur milieu, où la mémoire se perpétue et l'imagination se ravive, assurant ainsi le renouvellement d'un monde où l'on n'a pas seulement l'impression de mourir ou de disparaître, mais aussi le sentiment intime de se recréer dans le recommencement perpétuel d'un verbe qui se fait chair, mais chair multiple, chair du monde, progéniture sans fin et sans identités, comme les appelants et les parlants de Guyotat, qui ne sont plus des personnages au sens propre mais de véritables personnae, à travers lesquelles sonne la voix des langues qui se multiplient, des langues qui pullulent et prolifèrent, qui font nombre et font du même coup que le monde abonde, même s'il nous manque terriblement.

L'entrelangue ne s'érige pas comme une statue dans l'histoire humaine, même si c'est pour en marquer la borne, la dernière borne telle une sorte de stèle; elle se dresse en une colonne d'air ou de vent, éphémère comme le souffle et le temps, fragile comme l'âme, monument vivant que la vie jette en bas de son socle à tout moment, tel le putain sur le parquet où il se ramasse en petit tas, de cendre et de poussière, puis se relève péniblement, dans l'érection de sa voix audessus des bruits du monde, à quoi elle se mêle si intimement, dans une longue étreinte qui peu à peu l'étouffe. La langue elle-même est logoclaste et l'image iconoclaste parce que l'homme souffre depuis toujours d'anthropoclasmie: il brise l'humain en lui, qui tombe en ange putain, dont la mise bas le sauve d'une mort certaine, comme la langue se sauve dans l'entrelangue, l'icône et l'idole dans les morceaux de marbre et de bronze qui jonchent l'histoire comme autant de membres en chair et en os où l'on renaît en tant qu'être, être parlant, être mortel, être fini et en morceaux. Une œuvre comme Progénitures nous fait entendre le bruit des voix et des images qui tombent dans les langues comme on entend à répétition le son des corps humiliés et asservis qui heurtent le sol où l'histoire humaine ne cesse de les jeter, de les projeter, 
et d'où remontent une entrevoix, une entrelangue, dans lesquelles on peut capter les signes vitaux d'une entrehumanité, ni morte ni vivante mais survivante ou surmourante, qui profère son chant comme un avenir qu'elle prophétise du même souffle:

[...] ce nouvel état humain [...] c'est aussi le verbe, le verbe libre, le verbe totalement libre du putain - d'autant plus libre qu'il sort d'un corps qui n'a rien à perdre - qui, alors, est perdu. Le verbe est un paradis perdu. ${ }^{29}$

\section{NOTES}

1. Explications. Entretiens avec Marianne Alphant, Paris, Éd. Léo Scheer, 2000, p. 16.

2. La Bible - Ézéchiel, trad. A. Chouraqui, Paris, Desclée de Brouwer, 1985, 3, 13-14, p. 936.

3. V. Novarina, La Chair de l'homme, Paris, P.O.L, 1994.

4. Explications, op.cit., p. 15.

5. Progénitures, Paris, Gallimard, 2000, p. 19-20.

6. Ibid., p. 57 .

7. Explications, op.cit., p. 11 .

8. Ibid., p. 14.

9. Ibid., p. 15

10. Ibid., p. 13.

11. Ibid., p. 14
12. Progénitures, op.cit., p. 12.

13. D. Klébaner, Soutine, le tourment flamboyant, Paris, Somogy, 2000, p. 1. Klébaner ajoute: «les torsions des figures de Soutine [on pourrait dire de Guyotat] sont ces splendeurs gutturales, ces nodosités de gorge, ces épaississements de la sève, ces sarments de sang où la vivacité de l'éclat n'apparaît sombre que parce qu'elle rend sensible à vue ce travail de torsion" (ibid.).

14. La Bible - Ézéchiel, op. cit., 3, 1-3, p.935-936.

15. Explications, op.cit., p. 32.

16. Tous sens du verbe latin pro-ferre: porter au devant.

17. Ibid., p. 30

18. Vivre, Paris, Denoël, 1984.

19. Explications, op.cit., p. 35.

20. Ibid., p. 35-36.

21. Ibid., p. 35 .

22. Ibid., p. 30.

23. S. Beckett, Fin de partie, Paris, Éd. de Minuit, 1957, p. 15.

24. Guyotat écrit pourtant: "Le "putain" est une figure que je ne peux ni ne veux réprouver ni admirer; je l'aime. Pourquoi je l'aime? parce qu'il est quelque chose d'insituable entre Dieu, l'Histoire, et ce qu'on connait de l'humanité; et tout simplement parce que c'est un absolu, chose rare par les temps qui courent». (Explications, op. cit., p. 19, c'est moi qui souligne) - un absolu déchu qui nous rehausse, tombant du bas vers le haut, dans l'entre, dans l'insituable, comme l'entrelangue qui n'a pas de socle, pas de sol, renversée qu'elle est avec les statues et les monuments de l'Histoire, dans un dernier geste, iconoclaste, de ce qui reste ici-bas d'humanité.

25. Fin de partie, op.cit., p. 15-16.

26. Explications, op.cit., p. 13

27. Ibid., p. 136.

28. Progénitures, op.cit., p. 806.

29. Explications, op.cit., p. 21. 